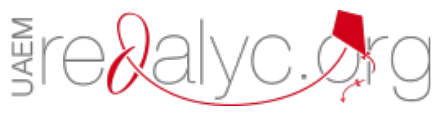

Centro Sur

ISSN: $2600-5743$

compasacademico@icloud.com

Grupo Compás

Ecuador

\title{
Cuerpo, sujeto y subjetividad
}

Sanabria Tovar, Manuel

Cuerpo, sujeto y subjetividad

Centro Sur, vol. 3, núm. 2, 2019

Grupo Compás, Ecuador

Disponible en: http://www.redalyc.org/articulo.oa?id=588861691001

Esta obra está bajo una Licencia Creative Commons Atribución-NoComercial-SinDerivar 4.0 Internacional. 


\section{Cuerpo, sujeto y subjetividad}

Centro Sur, vol. 3, núm. 2, 2019

Grupo Compás, Ecuador

Recepción: 10 Enero 2017

Aprobación: 12 Septiembre 2018

Redalyc: http://www.redalyc.org/ articulo.oa?id=588861691001

\section{BY-NC-ND}

\author{
Body, subject and subjectivity \\ Manuel Sanabria Tovar masatovar@gmail.com \\ Universidad Uniminuto de Dios centro Girardot, Colombia \\ (1D) http://orcid.org/0000-0003-3770-0230
}

\begin{abstract}
Resumen: El presente artículo presenta las reflexiones que el autor realiza sobre los conceptos de cuerpo, sujeto y subjetividad a partir de los desarrollos teóricos emprendidos por diferentes disciplinas de las ciencias sociales y, entre estas, por la psicología. Se muestra cómo el pensamiento se ha movilizado desde las tradicionales perspectivas dicotómicas propias de la modernidad hacia miradas más integradoras y articuladoras que vienen surgiendo en el marco de los llamados paradigmas emergentes que impactan hoy los desarrollos epistemológicos y metodológicos que orientan la producción de conocimiento sobre los diferentes objetos que abordan las investigaciones contemporáneas. No obstante esta apertura de la mirada no implica que las formas tradicionales de producción de conocimiento sobre el cuerpo, sujeto y subjetividad estén superadas, tampoco anuncian univocidades conceptuales, por el contrario el prisma del entendimiento es cada vez más complejo en el orden de la explicación, la comprensión y el abordaje de las realidades humanas.
\end{abstract}

Palabras clave: Corporeidad, cuerpo, subjetividad, sujeto.

Abstract: This article presents the reflections that the author makes about the concepts of body, subject and subjectivity from the theoretical developments undertaken by different disciplines of the social sciences and, among these, by psychology. It shows how thought has been mobilized from the traditional dichotomous perspectives of modernity towards more integrating and articulating perspectives that are emerging within the framework of the so-called emerging paradigms that impact today the epistemological and methodological developments that guide the production of knowledge about different objects that address contemporary research. However, this opening of the gaze does not imply that the traditional ways of producing knowledge about the body, subject and subjectivity are overcome, neither do they announce conceptual univocities, on the contrary the prism of understanding is increasingly complex in the order of explanation, understanding and approaching human realities. Keywords: Corporeity, body, subjectivity, subject.

\section{INTRODUCCIÓN}

La revisión de literatura científica publicada a partir de los estudios del cuerpo y la subjetividad muestra una producción cada vez más amplia. Estos estudios se situaban en sus inicios desde una perspectiva sociológico - antropológica, que cada vez ha trascendido más a ser considerados como objetos de investigación en los campos de los estudios culturales, literarios, históricos, filosóficos, psicológicos y artísticos. Ello ha permitido mirar el cuerpo y la subjetividad desde otros lugares que toman distancia de la tradicional óptica biomédica, para atender a sus implicaciones simbólicas enraizadas en diversas configuraciones de subjetividades.

Lo anterior ha permitido comprender el cuerpo inscrito en una compleja red simbólica cuyo sentido está imbricado en el tejido social por 
el que transita sumergido en un océano de significados que se manifiestan en las relaciones sociales.

Estas nuevas perspectivas conducen a la necesidad de entender el sujeto y el cuerpo como unidad que fue fragmentada en el pensamiento cartesiano. Esta unidad exige un esfuerzo por articular lo biológico, lo social, lo psicológico y lo cultural del sujeto y del cuerpo de la persona en un bucle constructivo. Ello requiere repensar las dicotomías instauradas en el pensamiento moderno que derivaron en la idea de un ser humano dual (cuerpo / mente) y que llevaron al énfasis otorgado a la racionalidad instrumental, naturalizando y universalizando tal perspectiva.

Los conceptos modernos de cuerpo y de sujeto están fundados en la subjetividad de la racionalidad y la productividad: "Desde lo propuesto por Descartes, en la perspectiva moderna lo fundamental es la razón, en tanto sujeto que piensa y objeto que debe ser pensado (perspectiva dicotómica)" (Ayala, M., Noreña, N., Sanabria Tovar, M., 2018, p. 30)

Sin embargo, conceptos como el de cuerpo y el de sujeto se constituyen de manera más frecuente en objetos de investigación en las diferentes ciencias sociales, sin poder afirmar el agotamiento de estos estudios.

$\mathrm{Al}$ realizar un recuento muy somero de los sentidos otorgados al concepto de cuerpo se encuentra que el uso cotidiano del término alude a algo extenso, limitado, perceptible y material (DRAE, 22a Edición).

El anterior sentido fue puesto de manifiesto por Planella (2002), quien al revisar las definiciones de los diccionarios encuentra predominante la concepción dualista del cuerpo y alude a lo material y físico, y en cuanto al cuerpo humano lo supone contrapuesto al alma.

No así sucede, afirma Planella (2002), en los estudios disciplinares desde la antropología y la pedagogía, en las que la idea de cuerpo va atada a la de persona considerando las dimensiones simbólicas, sociales y culturales, lo cual generó distinciones como cuerpo-objeto, cuerpo objetivado, físico, material, organismo (körper) y cuerpo-sujeto, cuerpo simbólico, subjetivo, sensible, vivencial, experiencial, intencional (leib).

Para nombrar este sentido que conlleva la consideración de la dimensión simbólica del cuerpo se acuñó el concepto de corporalidad. No obstante, al consultar el diccionario, la corporalidad se define como "cualidad de corporal, cosa corporal", donde lo corporal es lo "perteneciente o relativo al cuerpo, especialmente al humano (DRAE, 22a Edición).

Si bien, el concepto de corporalidad es un intento por dar cuenta de la dimensión simbólica del cuerpo, no incluye otras dimensiones implicadas en el cuerpo "leib" como son la dimensión subjetiva, existencial y relacional. Esto condujo a la necesidad de acuñar el concepto de corporeidad. En consecuencia, la corporalidad es condición material y de posibilidad de la corporeidad, constituyendo dos aspectos diferentes pero interrelacionados recíprocamente por la condición de seres encarnados (Merleau-Ponty, 1985). 


\section{MATERIALES Y MÉTODOS}

Por su parte, Zubiri (1987) precisa que dado que el ser humano se manifiesta con su cuerpo y a través de su cuerpo, la corporeidad constituye "la vivenciación del hacer, sentir, pensar y querer". Esto es, el concepto de corporeidad solo es atribuible al ser humano lo que permite diferenciarlo del concepto de cuerpo como realidad física al resaltar lo singular de lo humano.

Si bien, el ser humano nace como cuerpo es a partir de este como va configurando su corporeidad a través de la acción (Wallon, 1987). La psiquiatría también realizó el intento de articular la corporeidad con la corporalidad acudiendo a los conceptos de esquema corporal y su vivenciación por medio de la imagen corporal (Pastor, 2002). El esquema corporal o representación más o menos consciente de nuestro cuerpo, equivale al de cenestesia (imagen espacial del cuerpo); la imagen del cuerpo o vivencia diferencial de nuestro propio cuerpo, da lugar a la practognosia o cuerpo vivido; y, la imagen corporal o representación mental vaga del cuerpo, está influenciada por factores biológicos, cognitivos, afectivos, normas sociales y culturales, entre otros (Guimón citado por Pastor, op.cit. p.62). No obstante, este intento articulador se enfoca en el cuerpo como movimiento, más cercano al concepto de corporalidad.

\section{RESULTADOS}

Por otra parte, la distinción establecida por García Bacca (1987) por las que el cuerpo puede ser entendido como algo definido, estable y permanente (como tema) y el cuerpo como evolución, dinamismo y progreso (el cuerpo como problema) concuerda con la antinomia instaurada desde el pensamiento filosófico de la antigüedad entre lo permanente y lo que fluye.

Se pueden enumerar otros conceptos derivados de las anteriores líneas de pensamiento, como el de cuerpo presente que Varela denomina como neurofenomenología, en un intento por superar el reduccionismo cognitivista y la explicación fenomenológica de los estudios sobre los estados de conciencia y la experiencia humana, desde una urdimbre entre lo experiencial y lo material, lo natural y lo trascendental, a través de la "enacción", para superar el dualismo racionalista mente-cuerpo instaurado por Platón y Descartes y sostenido por Kant.

Más recientemente, se ha venido considerando la reflexión sobre el control político de la corporeidad, atendiendo a la acción política que se ejerce sobre el cuerpo con el propósito de controlar los comportamientos de los actores. El supuesto del que se parte es que el orden político se produce conjuntamente con el orden corporal. Así, un profesional o experto no dialoga solamente desde su saber disciplinar, sino con su cuerpo que los expresa y, al mismo tiempo, los cubre, puesto que las relaciones y las comunicaciones que se establecen en diferentes ámbitos son interacciones entre cuerpos que vehiculizan símbolos, cuerpos en 
dialogo incesante (Sanabria y Sanabria, 2008). El cuerpo es entonces la "dimensión del sujeto que posibilita la socialización, la encarnación y la corporeización del sujeto en el mundo" (Planella, 2002), dicho de otro modo, su subjetivación, en tanto metáforas que permean las sociedades en las diferentes épocas, produciendo diversos modelos corporales como mercancía, lenguaje, dominación, institucional, pragmático o productivo, resistencia, entre otros (Planella 2002).

Hasta aquí se ha expuesto de manera no exhaustiva la dinámica del pensamiento sobre el cuerpo y sus implicaciones para entender el sujeto y su proceso de subjetivación. A continuación se pretende abordar las formas como se viene entendiendo el concepto de subjetividad.

Los sentidos construidos sobre el concepto de subjetividad no son homogéneos. Se encuentra que, igual que el abordaje del cuerpo, hay posturas instaladas en el pensamiento dualista. En esta se ubican los autores que consideran la producción de la subjetividad como un proceso constituido primordialmente mediante la internalización de la realidad social, lo que presupone dos momentos separados como son los procesos mentales de carácter interno y la realidad externa. Así, la subjetividad es la resultante de la interiorización de lo social a partir de la facultad intrapsíquica del sujeto.

Otra perspectiva, sitúa la subjetividad en el espacio de lo histórico, cultural y social. Se distancia de la dicotomía clásica cartesiana entre cuerpo y razón, el adentro y el afuera. Desde allí, la subjetividad se inscribe en la escena de la dialéctica persona - sociedad y no como un procedimiento de interiorización en la dicotomía sujeto - objeto.

Las anteriores dos miradas sobre la subjetividad contienen distancias tanto de carácter epistemológico, en cuanto al lugar del conocimiento producido sobre la subjetividad, y de carácter metodológico al considerar la forma apropiada y pertinente para producir dicho conocimiento.

Diversos autores desde diferentes disciplinas como la sociología, la filosofía y la psicología, se han interesado por el estudio de la subjetividad, como Touraine, Bauman, Ferraroti, Deleuze, González Rey, Guattari y Rolnik. De estos autores el psicólogo cubano Fernando González Rey y el psicoanalista y filósofo Félix Guattari, muy prolíficos en esta temática, marcan dos perspectivas que pueden ser complementarias para la comprensión de la complejidad de la producción de conocimiento sobre la subjetividad.

Para González Rey (2011) la predominancia de la postura psicologicista sobre la persona humana ha entendido el individuo como una especie de respuesta mental ante los estímulos de su entorno. Su relación subjetiva con el mundo de la vida responde a un fenómeno intrapsíquico, aislado de alguna manera. De ahí que el sujeto es considerado como una especie de recipiente al que su entorno va llenando de información como dispositivo que le provee de las subjetividades para ser aplicadas en su cotidianidad. Visto así, la persona está enclavada en el soliloquio de su mente, en un determinismo del que apenas podrá librarse. Y ello tendría su explicación en todo el andamiaje positivista de la psicología tradicional que no ha logrado superar su vacío ontológico. 
La subjetividad de ninguna manera corresponde a un mundo de lo interior y otro de lo objetivo para ser internalizado por el sujeto, afirma González Rey (2000, pág. 28): "La subjetividad, queremos insistir, no se interioriza, se constituye mediante un proceso en que lo social actúa como instancia subjetiva, no como instancia objetiva desprovista de subjetividad".

Tal consideración de un mundo objetivo de la realidad social fuera de nosotros ha sido reproducida en las diversas instancias e instituciones sociales, al punto de ser naturalizada, como afirma Tomás Ibáñez (1994, p. 157 y 158):

Es probable, por ejemplo, que algunas personas no dudarían en afirmar que la realidad tiene propiedades objetivas que podría ser descritas, .... Por un observador culto. Otras por el contrario aseverarán que la realidad presenta una serie de propiedades que, aun siendo 'realmente' constitutivas de la misma, no dejan de ser, sin embargo, absolutamente subjetivas. Son propiedades que conforman la realidad objetiva pero que resultan de las actividades cognitivas,.... de las actividades simbólicas desarrolladas por los individuos

Por supuesto que nacemos a una realidad en la que se han cristalizado formas de organizarse, de relacionarse y hasta de aniquilarse; a un mundo con su propia historia, culturas, ritualidades, poderes, micropoderes, deseos e ideologías. La llegada a ese mundo tiene entonces unos precedentes de significado supremo por cuanto al entrar en contacto con este se inicia un proceso dialéctico para transformarlo o reproducirlo, aun sin suficiente consciencia de ello, del papel de agentes activos que tenemos.

La subjetividad, queremos insistir, no se interioriza: se constituye mediante un proceso en que lo social actúa como instancia subjetiva, no como instancia objetiva desprovista de subjetividad. Toda situación social objetiva se expresa con sentido subjetivo en las emociones y procesos significativos que se producen en los protagonistas de esas situaciones" (González Rey, 2000).

El mundo de la vida es pues una construcción permanente en el que participan la persona y los grupos sociales de manera dinámica, tensionante, dialéctica; al tenor de dichas relaciones igualmente se van configurando las subjetividades, que le dan sentido a esa realidad, se establecen deseos compartidos, emocionalidades, formas de simbolizar y actuar lo social.

Por supuesto que lo anterior no implica que el individuo pierda su carácter singular, que se diluya en lo social; o viceversa que sea lo social que se plegue por completo a un determinismo del sujeto. Ante todo constituye, como se ha venido insistiendo de una relación estrecha, inseparable, dialéctica, tensionante en cuyos espacios se configuran las subjetividades y se constituyen las subjetivaciones.

La subjetivación, como se ha expuesto no es un fenómeno de polos opuestos: individuo - grupos sociales, se trata de una relación necesaria, codependiente y dialéctica. En este sentido, Guattari y Rolnik proponen el concepto de "agenciamiento colectivo de enunciación", frente al 
de instancias psíquicas de Freud, que no corresponde a una entidad individual ni social predeterminada.

\section{CONCLUSIONES}

Se puede afirmar que el abordaje que Guattari et alt. (2006) realizan para dar cuenta de la subjetividad, expresa un intento por entender la forma como es constituida:

La subjetividad es producida por agenciamientos de enunciación. Los procesos de subjetivación o de semiotización no están centrados en agentes individuales (en el funcionamientode instancias intrapsíquicas, egoicas, microsociales), ni en agentes grupales. Esos procesos son doblemente descentrados... desde las más variadas vertientes, dinámicas, relaciones y espacios sociales como son los sistemas económicos, sociales, tecnológicos, icónicos, ecológicos, etológicos, de medios de comunicación de masas; así mismo se constituye la subjetividad mediante los sistemas de percepción, de sensibilidad, de afecto, de deseo, ideas, sistemas de inhibición y de automatismo, sistemas corporales, orgánicos, biológicos, fisiológicos.

Lo expuesto hasta aquí, desde estos autores, permite afirmar que en cada período histórico de la humanidad se han conformado diversas formas de relaciones sociales que se han cristalizado en formas de producción que definen a las sociedades llamadas primitivas, esclavistas, capitalistas y socialistas, en las que se han configurado sus propias subjetividades. En cada una de ellas se han modelado sujetos que responden a los intereses y necesidades de esas relaciones sociales, la mayor de las veces de sujeción, de adaptación al sistema, de lo cual la persona no es en la mayoría de las veces consciente.

Ello porque los modos de producción de subjetividades son cada vez más refinados, más sutiles, insertándose de forma más amplia en las diversas territorialidades por las que transita el sujeto como la familia, la escuela, las instituciones, el mercado, las comunicaciones, que se expresan como deseos y necesidades.

Es importante rescatar de lo que plantea González Rey en relación con la persona sujetada, que en cada persona hay una luz de resistencia que le permite asumir nuevas configuraciones en orden a su subjetivación, un nuevo sentido subjetivo de su vida que para González Rey es el espacio de relación inseparable de lo simbólico y lo emocional donde uno generalmente evoca al otro sin ser su causa (Rey, 2000).

En el mismo sentido afirman Guattarí et alt. (2006, p. 29)

A esa máquina de producción de subjetividad opondría la idea de que es posible desarrollar modos de subjetivación singulares, aquello que podríamos llamar «procesos de singularización»: una manera de rechazar todos esos modos de codificación preestablecidos, todos esos modos de manipulación y de control a distancia, rechazarlos para construir modos de sensibilidad, modos de relación con el otro, modos de producción, modos de creatividad que produzcan una subjetividad singular. 
Como se aprecia en lo anteriormente expuesto, la temática sobre la subjetividad además de ser compleja es abundante, por lo que aquí solamente se han reflexionado algunos aspectos, a nuestro juicio cruciales y sustanciales, sin pretender agotar ni saldar la discusión, al contrario se pretende producir referentes que provoquen diálogos desde el disenso o el consenso, que contengan la diversidad de matices que permitan ampliar la comprensión de los conceptos abordados en el marco de las ciencias sociales y de la psicología en particular.

\section{Referencias}

Ayala, M., Noreña, N., Sanabria Tovar, M (2018). Incursiones del cuerpo del maestro en el territorio educativo. En: Dalila, A. (Ed.) Educación e investigación Comprensiones desde la epistemología y el saber. Bogotá: Universidad Santo Tomás

DRAE. (2012).Diccionario de la Real Academia Española. Recuperado de http://www.rae.es/recursos/diccionarios/drae

García Bacca, J, (1987). Elogio de la técnica. Barcelona, España: Anthropos.

González Rey, F. (2011). Entrevista. Brasil: Blogger. Recuperado de http://ladesuece.blogspot.com/2011/05/subjetividadeentevista-comfernando.html

González Rey. F (2000). Investigación cualitativa en psicologia, rumbos y desafios. México: Internacional Thomson Editores

Guattari. F. y Rolnik. S. (2006). Micropolitica. Cartografía del deseo. Madrid. España: Traficantes de sueños.

Marcel, G (2002). El misterio del ser. Málaga, España: Autores Cristianos.

Merleau-Ponty, M (1985). Fenomenología de la percepción. Barcelona, España: Planeta-Agostini.

Pastor P, J. L (2002). Fundamentación conceptual para una intervención psicomotriz en Educación Física. Barcelona, España: INDE.

Planella, J (2002). Cuerpo, cultura y educación. Madrid, España: Desclèe de Brouwer.

Sanabria, M. y Sanabria, H. (2008). Hermenéutica de las formas de construcción del cuerpo desde la pedagogía (Maestría). Universidad Pedagógica Nacional - CINDE. Bogotá, Colombia

Ibáñez, T. (1994). Psicología Social Construccionista. México: Universidad de Guadalajara.

Wallon, H. (1987). Del acto al pensamiento. Barcelona, España: Paidós.

Zubiri, X. (1987). Sobre el hombre. Madrid: Alianza. 